\title{
The rate of convergence on fractional power dissipative operator on some sobolev type spaces
}

\author{
CAO Zhen-bin WANG Meng
}

\begin{abstract}
In [3], Chen, Deng, Ding and Fan proved that the fractional power dissipative operator is bounded on Lebesgue spaces $L^{p}\left(\mathbb{R}^{n}\right)$, Hardy spaces $H^{p}\left(\mathbb{R}^{n}\right)$ and general mixed norm spaces, which implies almost everywhere convergence of such operator. In this paper, we study the rate of convergence on fractional power dissipative operator on some sobolev type spaces.
\end{abstract}

\section{$\S 1 \quad$ Introduction}

We consider the fractional power dissipative equation

$$
\left\{\begin{array}{lrl}
u_{t}+(-\Delta)^{\alpha} u=0, & (x, t) & \in \mathbb{R}^{n} \times \mathbb{R}, \\
u(x, 0)=f(x), & x & \in \mathbb{R}^{n},
\end{array}\right.
$$

where $n \geq 2, \alpha>0, f: \mathbb{R}^{n} \rightarrow \mathbb{C}$ is the given initial data, and $\Delta=\sum_{i=1}^{n} \frac{\partial^{2}}{\partial x_{i}^{2}}$ is the Laplace operator. This interesting equation instantiates the heat equation if $\alpha=1$ and the Poisson's equation if $\alpha=1 / 2$. The solution of the above equation can be written by

$$
u(x, t)=e^{-t(-\Delta)^{\alpha}} f(x)=\left(e^{-t|\xi|^{2 \alpha}} \widehat{f}\right)^{\vee}(x),
$$

where $\widehat{f}$ is the Fourier transform of $f$, and $f^{\vee}$ is the inverse Fourier transform of $f$. We can also write $u(x, t)$ as a convolution operator:

$$
u(x, t)=K_{t}^{\alpha} * f(x),
$$

where

and

$$
K_{t}^{\alpha}(x)=\frac{1}{t^{\frac{n}{2 \alpha}}} K^{\alpha}\left(\frac{x}{t^{\frac{1}{2 \alpha}}}\right),
$$

$$
K^{\alpha}(x)=\int_{\mathbb{R}^{n}} e^{-|\xi|^{2 \alpha}} e^{2 \pi i x \cdot \xi} d \xi .
$$

In [10], Miao, Yuan and Zhang proved that $K^{\alpha}(x)$ satisfies that

$$
\left|K^{\alpha}(x)\right| \lesssim(1+|x|)^{-(n+2 \alpha)},
$$

Received: 2019-09-08. Revised: 2020-01-09.

MR Subject Classification: 42B25, 42B30, 42B35.

Keywords: convergence speed, dissipative operator, sobolev type spaces.

Digital Object Identifier(DOI): https://doi.org/10.1007/s11766-021-3901-8.

Supported by NSFC(11771388).

(C) The Author(s) 2021. 
for all $\alpha>0$, which immediately implies $e^{-t(-\Delta)^{\alpha}} f$ is bounded on Lebesgue spaces $L^{p}\left(\mathbb{R}^{n}\right)$ for $1 \leq p<\infty$. Also, by the multiplier theorem of Calderón and Torchinsky [1], $e^{-t(-\Delta)^{\alpha} f}$ is bounded on Hardy spaces $H^{p}\left(\mathbb{R}^{n}\right)$ for all $0<p<\infty$. We can see [3] on the boundedness of $e^{-t(-\Delta)^{\alpha}} f$ on other spaces. Also we can see $[6,8-11,15]$ on the fractional power dissipative equations with different potentials.

For $\lambda>0$, let $I_{-\lambda}$ denote the Riesz potential, i.e.

$$
I_{-\lambda}(f)=\left(|\xi|^{\lambda} \widehat{f}(\xi)\right)^{\vee} .
$$

Let $H^{p}\left(\mathbb{R}^{n}\right)$ denote the Hardy space, which is defined by

$$
\|f\|_{H^{p}\left(\mathbb{R}^{n}\right)}=\left\|\sup _{t>0}\left|\left(P_{t} * f\right)(x)\right|\right\|_{L^{p}\left(\mathbb{R}^{n}\right)},
$$

where $P$ is the Poisson kernel. A basic result is that $L^{p}\left(\mathbb{R}^{n}\right)$ and $H^{p}\left(\mathbb{R}^{n}\right)$ are equivalent when $p>1$. We further introduce the atomic decomposition: if $g \in H^{p}\left(\mathbb{R}^{n}\right)$, then

$$
g=\sum_{j} c_{j} a_{j}, \quad\|g\|_{H^{p}\left(\mathbb{R}^{n}\right)}^{p} \sim \sum_{j}\left|c_{j}\right|^{p}<\infty,
$$

where each $a_{j}$ is a $(p, 2)$-atom. Here $a$ is a $(p, 2)$-atom if there exists a cube $Q$ such that

(1) $a$ is supported in $Q$,

(2) $\|a\|_{L^{2}} \leq|Q|^{1 / 2-1 / p}$,

(3) $\int x^{\gamma} a(x) d x=0$ for all $\gamma$ with $|\gamma| \leq[n / p-n]$,

where $[x]$ means the largest integer that is not more than $x$. In the following argument, we mainly discuss the Sobolev type spaces $I_{-\lambda}\left(H^{p}\right)\left(\mathbb{R}^{n}\right) . I_{-\lambda}\left(H^{p}\right)\left(\mathbb{R}^{n}\right)$ is defined as the space of all $f$ satisfying $I_{-\lambda} f \in H^{p}\left(\mathbb{R}^{n}\right)$. For such function $f$, we define

$$
\|f\|_{I_{-\lambda}\left(H^{p}\right)\left(\mathbb{R}^{n}\right)}=\left\|I_{-\lambda}(f)\right\|_{H^{p}\left(\mathbb{R}^{n}\right)} .
$$

This space is the classical homogeneous Sobolev spaces for $p \geq 1$ and the Hardy-Sobolev spaces for $0<p \leq 1$ (see $[7,14])$.

We have already known the fractional power dissipative operator and related maximal operator are bounded on $H^{p}\left(\mathbb{R}^{n}\right)$, which implies for each $f \in H^{p}\left(\mathbb{R}^{n}\right), \lim _{t \rightarrow 0} e^{-t(-\Delta)^{\alpha}} f=f$ a.e. Then we ask: if $f$ has more regularity, can we obtain better estimates? Our result is the following.

Theorem 1.1. Let $\alpha>0, p>0$ and $0 \leq \lambda<2 \alpha$. If $f \in I_{-\lambda}\left(H^{p}\right)\left(\mathbb{R}^{n}\right)$, then

$$
e^{-t(-\Delta)^{\alpha}} f(x)-f(x)=o\left(t^{\frac{\lambda}{2 \alpha}}\right) \quad \text { a.e. as } t \rightarrow 0 \text {. }
$$

Firstly we consider the case for $\lambda=0$, by the method from [13], Theorem 1.1 can be reduced to

$$
\left\|\sup _{t>0}\left|e^{-t(-\Delta)^{\alpha}} f-f\right|\right\|_{L^{p}\left(\mathbb{R}^{n}\right)} \lesssim\|f\|_{H^{p}\left(\mathbb{R}^{n}\right)} .
$$

This result is correct since the fractional power dissipative operator is bounded on $H^{p}\left(\mathbb{R}^{n}\right)$ and

$$
\|f\|_{L^{p}\left(\mathbb{R}^{n}\right)}=\left\|\lim _{t \rightarrow 0}\left(e^{-t|x|^{2}} * f\right)\right\|_{L^{p}\left(\mathbb{R}^{n}\right)} \leq\|f\|_{H^{p}\left(\mathbb{R}^{n}\right)}
$$

holds for all $0<p<\infty$.

From now on, let $0<\lambda<2 \alpha$. We repeat the method of [13], then Theorem 1.1 can be reduced to the following result. 
Theorem 1.2. Let $\alpha>0, p>0$ and $0<\lambda<2 \alpha$. If $f \in I_{-\lambda}\left(H^{p}\right)\left(\mathbb{R}^{n}\right)$, then

$$
\left\|\sup _{t>0} t^{-\frac{\lambda}{2 \alpha}}\left|e^{-t(-\Delta)^{\alpha}} f-f\right|\right\|_{L^{p}\left(\mathbb{R}^{n}\right)} \lesssim\|f\|_{I_{-\lambda}\left(H^{p}\right)\left(\mathbb{R}^{n}\right)} .
$$

We will prove this theorem in section 2. We decompose the integral in Theorem 1.2 into two parts. As the argument in Chen [2], the estimate of kernel on each part is core of our proof, which is left at Lemma 2.2 .

Throughout this paper, $A \lesssim B$ means that there exists a constant $C>0$ independent of all essential variables such that $A \leq C B$, and $A \sim B$ means $A \lesssim B$ and $B \lesssim A$. The space of all infinitely differentiable functions on $\mathbb{R}^{n}$ is denoted by $\mathcal{C}^{\infty}\left(\mathbb{R}^{n}\right)$. The space of $\mathcal{C}^{\infty}$ functions with compact support is denoted by $\mathcal{C}_{0}^{\infty}\left(\mathbb{R}^{n}\right)$. The space of $\mathcal{C}^{\infty}$ functions with all derivatives rapidly decreasing is denoted by $\mathcal{S}\left(\mathbb{R}^{n}\right)$. Let $\beta=\left(\beta_{1}, \ldots, \beta_{n}\right)$ where $\beta_{j} \in \mathbb{Z}$ and $\beta_{j} \geq 0$ for $0 \leq j \leq n$, $\partial^{\beta} f$ means the derivative $\partial^{\beta_{1}} \ldots \partial^{\beta_{n}} f$.

\section{$\S 2 \quad$ Proof of Theorem 1.2}

There is a standard result of Stein $[12]$ on $H^{p}\left(\mathbb{R}^{n}\right)$. We state it here.

Lemma 2.1. [12] Let $0<p \leq 1$. Suppose that a function $\zeta$ vanishes at $\infty$ and satisfies

$$
\left|\partial^{\beta} \zeta(x)\right| \lesssim(1+|x|)^{-A}
$$

for $|\beta|=[n(1 / p-1)]+1$ and $A>n / p$. Then there is a constant $C>0$, for any $f \in H^{p}\left(\mathbb{R}^{n}\right)$,

$$
\left\|\sup _{R>0}\left|f * \zeta_{R}\right|\right\|_{L^{p}\left(\mathbb{R}^{n}\right)} \leq C\|f\|_{H^{p}\left(\mathbb{R}^{n}\right)},
$$

where $\zeta_{R}(x)=R^{-n} \zeta(x / R)$ for $R>0$.

Write

$$
\begin{aligned}
& t^{-\frac{\lambda}{2 \alpha}}\left|e^{-t(-\Delta)^{\alpha}} f-f\right| \\
= & t^{-\frac{\lambda}{2 \alpha}}\left|\int_{\mathbb{R}^{n}}\left(e^{-t|\xi|^{2 \alpha}}-1\right) \widehat{f}(\xi) e^{2 \pi i \xi \cdot x} d \xi\right| \\
= & \left.t^{-\frac{\lambda}{2 \alpha}}\left|\int_{\mathbb{R}^{n}}\left(e^{-\left|t^{\frac{1}{2 \alpha}} \xi\right|^{2 \alpha}}-1\right) \frac{1}{|\xi|^{\lambda}} \widehat{f}(\xi)\right| \xi\right|^{\lambda} e^{2 \pi i \xi \cdot x} d \xi \mid \\
= & \left.\left|\int_{\mathbb{R}^{n}}\left(e^{-\left|t^{\frac{1}{2 \alpha}} \xi\right|^{2 \alpha}}-1\right) \frac{1}{\left|t^{\frac{1}{2 \alpha}} \xi\right|^{\lambda}} \widehat{f}(\xi)\right| \xi\right|^{\lambda} e^{2 \pi i \xi \cdot x} d \xi \mid \\
= & \left|I_{-\lambda}(f) *\left(g^{\alpha, \lambda}\right)_{t}\right|,
\end{aligned}
$$

where

To prove Theorem 1.2, it suffices to show

$$
g^{\alpha, \lambda}(x)=\int_{\mathbb{R}^{n}}\left(e^{-|\xi|^{2 \alpha}}-1\right) \frac{1}{|\xi|^{\lambda}} e^{2 \pi i \xi \cdot x} d \xi
$$

$$
\left\|\sup _{t>0}\left|I_{-\lambda}(f) *\left(g^{\alpha, \lambda}\right)_{t}\right|\right\|_{L^{p}} \lesssim\left\|I_{-\lambda}(f)\right\|_{H^{p}} .
$$

Set $h=I_{-\lambda}(f)$, the above inequality is reduced to

$$
\left\|\sup _{t>0}\left|h *\left(g^{\alpha, \lambda}\right)_{t}\right|\right\|_{L^{p}} \lesssim\|h\|_{H^{p}}
$$


Let $\phi_{0}, \phi_{\infty} \in \mathcal{C}^{\infty}\left(\mathbb{R}^{n}\right)$ be radial functions satisfying the following conditions:

$$
\phi_{0}(\xi)=\left\{\begin{array}{ll}
1 & |\xi| \leq 1 / 2, \\
0 & |\xi| \geq 1 ;
\end{array} \quad \phi_{\infty}(\xi)= \begin{cases}0 & |\xi| \leq 1 / 2, \\
1 & |\xi|>1 ;\end{cases}\right.
$$

and $\phi_{0}+\phi_{\infty}=1$. Then we divide the integral $g^{\alpha, \lambda}$ to $g_{0}^{\alpha, \lambda}$ and $g_{\infty}^{\alpha, \lambda}$ :

and

$$
g_{0}^{\alpha, \lambda}(x)=\int_{\mathbb{R}^{n}} \phi_{0}(\xi)\left(e^{-|\xi|^{2 \alpha}}-1\right) \frac{1}{|\xi|^{\lambda}} e^{2 \pi i \xi \cdot x} d \xi
$$

$$
g_{\infty}^{\alpha, \lambda}(x)=\int_{\mathbb{R}^{n}} \phi_{\infty}(\xi)\left(e^{-|\xi|^{2 \alpha}}-1\right) \frac{1}{|\xi|^{\lambda}} e^{2 \pi i \xi \cdot x} d \xi .
$$

Firstly we consider $g_{0}^{\alpha, \lambda}$. Via the similar method of Lemma 2.1 in [4] (we also can see [5]), one concludes: for $0<\lambda<2 \alpha$, all $\beta=\left(\beta_{1}, \ldots, \beta_{n}\right)$ with $\beta_{j} \geq 0$ for $0 \leq j \leq n$,

When $p>1$, by (1),

$$
\left|\partial^{\beta} g_{0}^{\alpha, \lambda}(x)\right| \lesssim \frac{1}{(1+|x|)^{n+|\beta|+2 \alpha-\lambda}} .
$$

$$
\left|g_{0}^{\alpha, \lambda}(x)\right| \lesssim \frac{1}{(1+|x|)^{n+2 \alpha-\lambda}}
$$

which is controlled by one integrable radially decreasing function due to $0<\lambda<2 \alpha$. Therefore

$$
\left\|\sup _{t>0}\left|h *\left(g_{0}^{\alpha, \lambda}\right)_{t}\right|\right\|_{L^{p}} \lesssim\|M(h)\|_{L^{p}} \lesssim\|h\|_{L^{p}} \sim\|h\|_{H^{p}} .
$$

Here we use that $L^{p}$ is equivalent to $H^{p}$ when $p>1$. When $0<p \leq 1$, by (1),

$$
\left|\partial^{\beta} g_{0}^{\alpha, \lambda}(x)\right| \lesssim \frac{1}{(1+|x|)^{n+|\beta|+2 \alpha-\lambda}} .
$$

Considering $n+|\beta|+2 \alpha-\lambda>\frac{n}{p}$ when $|\beta|=\left[n\left(\frac{1}{p}-1\right)\right]+1$, by Lemma 2.1,

$$
\left\|\sup _{t>0}\left|h *\left(g_{0}^{\alpha, \lambda}\right)_{t}\right|\right\|_{L^{p}} \lesssim\|h\|_{H^{p}}
$$

Next we consider $g_{\infty}^{\alpha, \lambda}$. We have the following estimate (proof can be seen in the following Lemma 2.2):

$$
\left|\partial^{\beta} g_{\infty}^{\alpha, \lambda}(x)\right| \lesssim \frac{1}{|x|^{n+|\beta|-\lambda+L}}
$$

holds for all $L \geq 0$ so that $n+|\beta|-\lambda+L \geq 0$. When $p>1$, by (2), $g_{\infty}^{\alpha, \lambda}$ is controlled by one integrable radially decreasing function, then

$$
\left\|\sup _{t>0}\left|h *\left(g_{\infty}^{\alpha, \lambda}\right)_{t}\right|\right\|_{L^{p}} \lesssim\|M(h)\|_{L^{p}} \lesssim\|h\|_{L^{p}} \sim\|h\|_{H^{p}} .
$$

When $0<p \leq 1$, by the atomic decomposition of $h, h=\sum_{j} \lambda_{j} a_{j}$, where each $a_{j}$ is a $(p, 2)$-atom and $\|h\|_{H^{p}}^{p} \sim \sum_{j}\left|\lambda_{j}\right|^{p}$. The properties of atom can be seen in section 1. Then

$$
\begin{aligned}
& \left\|\sup _{t>0}\left|h *\left(g_{\infty}^{\alpha, \lambda}\right)_{t}\right|\right\|_{L^{p}}^{p} \\
\leq & \left\|\sum_{j}\left|\lambda_{j}\right| \sup _{t>0}\left|a_{j} *\left(g_{\infty}^{\alpha, \lambda}\right)_{t}\right|\right\|_{L^{p}}^{p} \\
\leq & \sum_{j}\left|\lambda_{j}\right|^{p}\left\|\sup _{t>0}\left|a_{j} *\left(g_{\infty}^{\alpha, \lambda}\right)_{t}\right|\right\|_{L^{p}}^{p} .
\end{aligned}
$$


So if for each atom $a$, we have

$$
\left\|\sup _{t>0}\left|a *\left(g_{\infty}^{\alpha, \lambda}\right)_{t}\right|\right\|_{L^{p}} \lesssim 1
$$

then

$$
\left\|\sup _{t>0}\left|h *\left(g_{\infty}^{\alpha, \lambda}\right)_{t}\right|\right\|_{L^{p}} \lesssim\left(\sum_{j}\left|\lambda_{j}\right|^{p}\right)^{1 / p} \sim\|h\|_{H^{p}} .
$$

We prove (3) in the last lemma. The proof of Theorem 1.2 is completed as all cases have been proved.

Finally, we give the proofs of (2) and (3).

Lemma 2.2. For $\lambda>0, \beta=\left(\beta_{1}, \ldots, \beta_{n}\right)$ with $\beta_{j} \geq 0$ for $0 \leq j \leq n$, we have

$$
\left|\partial^{\beta} g_{\infty}^{\alpha, \lambda}(x)\right| \lesssim \frac{1}{|x|^{n+|\beta|-\lambda+L}}
$$

holds for all $L \geq 0$ so that $n+|\beta|-\lambda+L \geq 0$.

Proof. Write

$$
g_{\infty}^{\alpha, \lambda}(x)=\int_{\mathbb{R}^{n}} \frac{e^{-|\xi|^{2 \alpha}}}{|\xi|^{\lambda}} \phi_{\infty}(\xi) e^{2 \pi i \xi \cdot x} d \xi-\int_{\mathbb{R}^{n}} \frac{1}{|\xi|^{\lambda}} \phi_{\infty}(\xi) e^{2 \pi i \xi \cdot x} d \xi
$$

We only estimate the second term that is denoted by $K^{\lambda}(x)$, then the first term can be treated by the same way.

As to $K^{\lambda}(x)$, we decompose it by the partition of unity,

$$
1=\eta(\xi)+\sum_{j=0}^{\infty} \delta\left(2^{-j} \xi\right)
$$

where $\eta$ and $\delta$ are all in $\mathcal{S}\left(\mathbb{R}^{n}\right)$, supp $\eta \subset\{x|| x \mid \leq 1\}$, supp $\delta \subset\{x|1 / 2 \leq| x \mid \leq 2\}$, so supp $\delta\left(2^{-j}\right) \subset\left\{x\left|2^{j-1} \leq\right| x \mid \leq 2^{j+1}\right\}$ for $j \geq 1$. Noting the support of $\phi_{\infty}$, we have

$$
K^{\lambda}(x)=\sum_{j=1}^{\infty} K_{j}^{\lambda}(x)
$$

where

We claim:

$$
K_{j}^{\lambda}(x)=\int_{\mathbb{R}^{n}} \frac{1}{|\xi|^{\lambda}} \delta\left(2^{-j} \xi\right) e^{2 \pi i \xi \cdot x} d \xi
$$

$$
\left|\partial^{\beta} K_{j}^{\lambda}(x)\right| \lesssim|x|^{-M} 2^{j(n+|\beta|-\lambda-M)}
$$

holds for all $M \geq 0$.

In fact, write

$$
(-2 \pi i x)^{\gamma} \partial^{\beta} K_{j}^{\lambda}(x)=\int_{\mathbb{R}^{n}} \partial_{\xi}^{\gamma}\left[(2 \pi i \xi)^{\beta} \frac{\delta\left(2^{-j} \xi\right)}{|\xi|^{\lambda}}\right] e^{2 \pi i \xi \cdot x} d \xi .
$$

Using the support of $\delta\left(2^{-j} \cdot\right)$, we obtain

$$
\left|x^{\gamma} \partial^{\beta} K_{j}^{\lambda}(x)\right| \lesssim \int_{\mathbb{R}^{n}} \delta\left(2^{-j} \xi\right)|\xi|^{|\beta|-\lambda-|\gamma|} d \xi \lesssim 2^{j(n+|\beta|-\lambda-|\gamma|)} .
$$

There is a basic fact: for $\gamma=\left(\gamma_{1}, \ldots, \gamma_{n}\right)$,

$$
|x|^{|\gamma|} \leq C_{n, \gamma} \sum_{|\beta|=|\gamma|}\left|x^{\beta}\right|
$$


holds for all $x \neq 0$. Since $\gamma$ is arbitrary, we take the supremum over all $\gamma$ with $|\gamma|=M$, then (5) implies (4).

Since $K^{\lambda}(x)=\sum_{j=1}^{\infty} K_{j}^{\lambda}(x)$, it suffices to estimate $\sum_{j=1}^{\infty}\left|\partial^{\beta} K_{j}^{\lambda}(x)\right|$. When $0<|x| \leq 1$, we divide it to two parts: the first part $2^{j} \leq|x|^{-1}$, the second part $2^{j}>|x|^{-1}$. For the first term, we use (4) when $M=0$ to get

$$
\sum_{2^{j} \leq|x|^{-1}}\left|\partial^{\beta} K_{j}^{\lambda}(x)\right| \leq \sum_{2^{j} \leq|x|^{-1}} 2^{j(n+|\beta|-\lambda)},
$$

which is $O\left(|x|^{-n-|\beta|+\lambda}\right)$ when $n+|\beta|-\lambda>0$, and $O\left(\log \left(|x|^{-1}\right)+1\right)$ when $n+|\beta|-\lambda \leq 0$. In either case we get the bound

$$
O\left(|x|^{-n-|\beta|+\lambda-L}\right)
$$

with the restrictions that $|x| \leq 1, L \geq 0$, and $n+|\beta|-\lambda+L \geq 0$. For the second term, we choose $M>n+|\beta|-\lambda$ in (4), then we get

$$
O\left(|x|^{-M}\right) \sum_{2^{j}>|x|^{-1}} 2^{j(n+|\beta|-\lambda-M)}=O\left(|x|^{-n-|\beta|+\lambda}\right),
$$

so the bound is $O\left(|x|^{-n-|\beta|+\lambda-L}\right)$ if $L \geq 0$. When $|x| \geq 1$, we choose $M>n+|\beta|-\lambda+L$, then from (4) we get the bound $|x|^{-M}$, which is $O\left(|x|^{-n-|\beta|+\lambda-L}\right.$ ) for every $L$.

Lemma 2.3. Suppose that $a$ is a $(p, 2)$-atom, then

$$
\left\|\sup _{t>0}\left|a *\left(g_{\infty}^{\alpha, \lambda}\right)_{t}\right|\right\|_{L^{p}} \lesssim 1
$$

Proof: We assume that supp $a \subset Q$, where $Q$ is a cube with the center of zero. Other cases are similar since this operator is a convolution operator (it commutes with translations). Then

$$
\begin{aligned}
& \left\|\sup _{t>0}\left|a *\left(g_{\infty}^{\alpha, \lambda}\right)_{t}\right|\right\|_{L^{p}}^{p} \\
= & \int_{\mathbb{R}^{n}} \sup _{t>0}\left|a *\left(g_{\infty}^{\alpha, \lambda}\right)_{t}\right|^{p} \\
= & \int_{2 Q} \sup _{t>0}\left|a *\left(g_{\infty}^{\alpha, \lambda}\right)_{t}\right|^{p}+\int_{(2 Q)^{c}} \sup _{t>0}\left|a *\left(g_{\infty}^{\alpha, \lambda}\right)_{t}\right|^{p} \\
= & I+I I .
\end{aligned}
$$

For $I$, since $g_{\infty}^{\alpha, \lambda}$ is controlled by one integrable radially decreasing function, $T(f)=\sup _{t>0} \mid f *$ $\left(g_{\infty}^{\alpha, \lambda}\right)_{t} \mid$ is bounded on $L^{2}$. Then

$$
\begin{aligned}
I & =\int_{2 Q}|T(a)|^{p} \leq\left(\int_{2 Q} 1\right)^{1-\frac{p}{2}}\left(\int_{2 Q} T(a)^{2}\right)^{\frac{p}{2}} \\
& \lesssim|Q|^{1-\frac{p}{2}}\|a\|_{L^{2}}^{p} \leq|Q|^{1-\frac{p}{2}}|Q|^{\frac{p}{2}-1}=1 .
\end{aligned}
$$

Next we start to estimate $I I$. Set $N=\left[n\left(\frac{1}{p}-1\right)\right]$. Considering the vanishing property of $a$, for some $\theta \in(0,1)$ such that

$$
\begin{aligned}
& \left|a *\left(g_{\infty}^{\alpha, \lambda}\right)_{t}\right| \\
= & \frac{1}{t^{n}}\left|\int_{Q} a(y) g_{\infty}^{\alpha, \lambda}\left(\frac{x-y}{t}\right) d y\right|
\end{aligned}
$$




$$
\begin{aligned}
& =\frac{1}{t^{n}}\left|\int_{Q} a(y)\left[g_{\infty}^{\alpha, \lambda}\left(\frac{x-y}{t}\right)-\sum_{|\beta| \leq N} \partial^{\beta} g_{\infty}^{\alpha, \lambda}\left(\frac{x}{t}\right) \frac{(-y / t)^{\beta}}{\beta !}\right] d y\right| \\
& =\frac{1}{t^{n}}\left|\int_{Q} a(y)\left[\sum_{|\beta|=N+1} \partial^{\beta} g_{\infty}^{\alpha, \lambda}\left(\frac{x-\theta y}{t}\right) \frac{(-y / t)^{\beta}}{\beta !}\right] d y\right| \\
& \lesssim \frac{1}{t^{n}} \int_{Q}|a(y)|\left|\frac{y}{t}\right|^{N+1} \frac{1}{\left|\frac{x}{t}\right|^{n+N-\lambda+L+1}} d y,
\end{aligned}
$$

where $|x-\theta y| \geq|x|-|y| \geq \frac{1}{2}|x|$ since $y \in Q$ and $x \in(2 Q)^{c}$, and the last line is due to Lemma 2.2. We choose $L=\lambda>0$, then

hence

$$
\left|a *\left(g_{\infty}^{\alpha, \lambda}\right)_{t}\right| \lesssim \frac{1}{|x|^{n+N+1}} \int_{Q}|a(y)||y|^{N+1} d y \lesssim \frac{|Q|^{1-\frac{1}{p}+\frac{N+1}{n}}}{|x|^{n+N+1}},
$$

$$
\begin{gathered}
I I=\int_{(2 Q)^{c}} \sup _{t>0}\left|a *\left(g_{\infty}^{\alpha, \lambda}\right)_{t}\right|^{p} \\
\lesssim \int_{(2 Q)^{c}} \frac{|Q|^{p-1+\frac{p(N+1)}{n}}}{|x|^{p(n+N+1)}} d x \\
\lesssim|Q|^{p-1+\frac{p(N+1)}{n}} \int_{(2 Q)^{c}} \frac{1}{|x|^{p(n+N+1)}} d x .
\end{gathered}
$$

Since $p(n+N+1)>n$ when $N=\left[n\left(\frac{1}{p}-1\right)\right]$,

$$
I I \lesssim|Q|^{p-1+\frac{p(N+1)}{n}}|Q|^{-p+1-\frac{p(N+1)}{n}}=1 .
$$

We finish the proof of this lemma.

Open Access This article is licensed under a Creative Commons Attribution 4.0 International License, which permits use, sharing, adaptation, distribution and reproduction in any medium or format, as long as you give appropriate credit to the original author(s) and the source, provide a link to the Creative Commons licence, and indicate if changes were made. The images or other third party material in this article are included in the articles Creative Commons licence, unless indicated otherwise in a credit line to the material. If material is not included in the articles Creative Commons licence and your intended use is not permitted by statutory regulation or exceeds the permitted use, you will need to obtain permission directly from the copyright holder. To view a copy of this licence, visit http://creativecommons.org/licenses/by/4.0/.

\section{References}

[1] A Calderón, A Torchinsky. Parabolic functions associated with a distribution, II, Advances in Math, 1977, 24(2): 101-171.

[2] J Chen. Weak type $(1,1)$ boundedness of Riesz transform on positively curved manifolds, Chinese Annals Math, Series B, 1992, 13(1): 1-5. 
[3] J Chen, Q Deng, Y Ding, D Fan. Estimates on fractional power dissipative equations in function spaces, Nonlinear Analysis, 2012, 75(5): 2959-2974.

[4] D Fan, F Zhao. Almost everywhere convergence of Bochner-Riesz means on some Sobolev type spaces, Ann Funct Anal, 2019, 10(1): 29-45.

[5] D Fan, F Zhao. Block-Sobolev Spaces and the Rate of Almost Everywhere Convergence of Bochner-Riesz Means, Constructive Approximation, 2017, 45(3): 391-405.

[6] Y Giga. Solutions for semilinear parabolic equations in $L^{p}$ and regularity of weak solutions of the Navier-Stokes system, Journal of Differential Equations, 1985, 62(2): 186-212.

[7] L Grafakos. Classical Fourier analysis, Graduate Texts in Math, Spinger, New York, 2012.

[8] H Matthias, P Jan. Heat kernels and maximal lpâlqestimates for parabolic evolution equations, Communications in Partial Differential Equations, 1997, 22(9-10): 1647-1669.

[9] C Miao, B Yuan, B Zhang. Strong solutions to the nonlinear heat equation in homogeneous Besov spaces, Nonlinear Analysis Theory Methods and Applications, 2007, 67(5): 1329-1343.

[10] C Miao, B Yuan, B Zhang. Well-posedness of the Cauchy problem for the fractional power dissipative equations, Nonlinear Analysis, 2008, 68(3): 461-484.

[11] C Miao, B Zang. The Cauchy problem for semilinear parabolic equations in Besov spaces, Houston Journal of Mathematics, 2004, 30(3): 829-878.

[12] E M Stein. Harmonic Analysis, Real Variable Methods, Orthogonality, and Oscillatory Integrals, Princeton University Press, Princeton, New Jersey, 1993.

[13] E M Stein, M H Taibleson, G Weiss. Weak type estimates for maximal operators on certain $H^{p}$ classes, Proceedings of the Seminar on Harmonic Analysis, 1981, 2(1): 81-97.

[14] R Strichartz. $H^{p}$ Sobolev spaces, Colloq Math, 1990, LX/LXI: 129-139.

[15] E Terraneo. Non-Uniqueness for a critical non-kunear heat equation, Communications in Partial Differential Equations, 2002, 27(1-2): 185-218.

Department of Mathematics, Zhejiang University, Hangzhou 310027, China.

Email: 11735002@zju.edu.cn, mathdreamcn@zju.edu.cn 